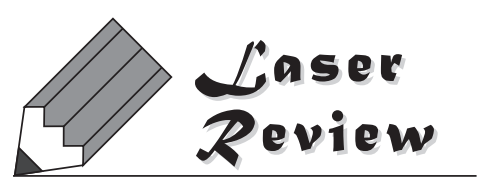

\title{
Ultrafast Laser Processing for Industrial Applications
}

\author{
Andreas OSTENDORF and Stella MARAGKAKI \\ Chair of Applied Laser Technology, Ruhr-University Bochum, Germany
}

(Received July 16, 2013)

\begin{abstract}
The development of ultrafast laser technologies within the last years created new perspectives, leading to the realization of new technologies and applications. In the automotive and mechanical industry the need for precision and high efficiency in a variety of applications make the ultrafast laser pulses an invaluable tool. Each of the studies presented in this review demonstrates the benefits of ultrafast laser pulses in industrial applications such as micro drilling of injection nozzles, lubrication reservoirs in laser honing and roll texturing.
\end{abstract}

Key Words: Ultrashort pulses, Drilling, Honing, Roll texturing

\section{Introduction}

Major advancements in the automotive and mechanical industry have been achieved during the last years by using ultrafast laser pulses. The main characteristic of ultrashort laser pulse ablation is the deposition of energy in a very short time period leading to rapid plasma formation and evaporation of the irradiated material. When applying short laser pulses to ablate metals, which typically have comparatively low melting temperatures and high conductivity, the absence of thermal diffusion results in very precise structures with generally no heat affected zones. Further benefit of using ultrashort pulses is the high efficiency of the ablation process compared to nanosecond ablation. Furthermore, low laser fluences, slightly above the ablation threshold, allow high-quality micro-processing and large area patterning without thermal or mechanical damage. However, for many practical applications a high processing speed is required. Thus, material processing with much higher laser fluences is attempted. However, drilling by high laser intensities has shown that some of the advantages of ultrafast pulses could be lost. In the high fluence regime a considerable amount of molten material can be generated, leading to stresses which can cause deformation in the material. ${ }^{1)}$ Precise drilling experiments have demonstrated the rapid elongation of the processing time when increasing the pulse duration. ${ }^{2}$ Therefore, the application of laser pulses in the sub-picosecond time scale does not only increase the precision, but also reduces the processing time. Potential application of these drilling tools is the machining of components for the automotive industry (e.g. injection nozzles), for the textile industry (e.g. spinnerets) and for the mechanical industry.

\section{Automotive industry}

\subsection{Drilling of diesel and fuel injectors}

In the field of automotive industry, the environmental protection and lower fuel consumption are the drivers of more precision in engine components. In order to improve combustion efficiency and to reduce pollutants, high-precision microholes are required for the next generation high pressure diesel fuel injection nozzles. Therefore, it is effective to optimize the size, pattern and accuracy of nozzle holes. ${ }^{3)}$ At present, drilling is a common method of micro-hole processing, for example mechanical drilling, long pulse laser drilling, electric discharge machining (EDM) drilling and ultrafast laser drilling. Compared to the conventional drilling methods, ultrafast laser pulses offer some notable advantages such as high precision without micro-cracks, high processing speed, high efficiency, absence of defects on the hole-edges. Furthermore, additional post-processing or special gas environment are not required. Therefore, ultrafast laser drilling has become a valuable tool for the manufacture of high-quality micro holes in metals.

Over the last decade, many investigations have been accomplished in ultrafast laser microdrilling of metals. In an early study by Kamlage et. al. on deep drilling of metals, ${ }^{4}$ holes in a $1 \mathrm{~mm}$ thick stainless steel plate were fabricated in air and in vacuum. Stainless steel is commonly used in automotive industries for the fabrication of fuel injectors due to its high hardness and the very good resistance to chemical erosion. The holes were drilled by $0.9 \mathrm{~mJ}, 150$ fs laser pulses focused in the surface (Fig. 1). Comparing the laser drilling in the atmospheric pressure and in vacuum $\left(10^{-3} \mathrm{mbar}\right)$, a reduction of the entrance hole diameter and at the same time an increase of the exit hole diameter with a growing laser pulse repetition rate were observed. Whereas, the holes drilled in vacuum have the same convergent shape regardless of the growing laser pulse repetition rate. More specifically, $100 \mu \mathrm{m}$ entrance
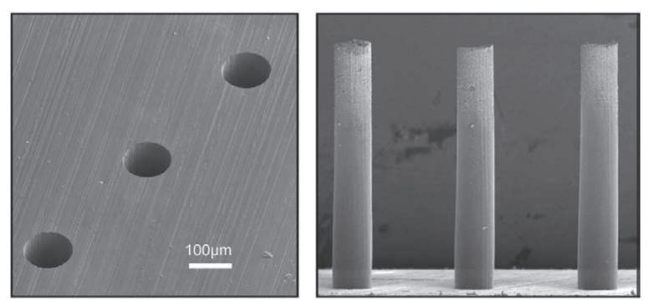

Fig. 1 Scanning electron microscope image of holes drilled in a $1 \mathrm{~mm}$ thick stainless steel plate with fs laser pulses. Kamlage et. al. ${ }^{4)}$ 
hole diameters and $40 \mu \mathrm{m}$ exit hole diameters were formed. A possible explanation for the reduction of the entrance hole diameter with increasing repetition rate is that, at constant atmospheric pressures, a lower gas particle density was caused by the high air temperature. The lower gas particle density in the focus region leads to better focused laser pulses with lower deformations. ${ }^{4)}$

In a recent study of Kling et. al., ${ }^{5)}$ the authors demonstrated femtosecond laser drilling of stainless steel using a trepanning head. The laser trepanning technique allows to improve the hole quality by moving the beam on a circular path relative to the target. In this way, the ablated material is removed easier due to the smaller size of the laser spot and the hole shape is less affected by the beam profile. As a result, a reduction of burr formation is observed. Trepanning heads are well known to provide a high-precision hole geometry. With the combination of trepanning head and femtosecond pulses (500 fs, 515 $\mathrm{nm}, 80 \mu \mathrm{J}$ and $10 \mathrm{kHz}$ ), there is a possibility of independent adjustment of the hole diameter and the inclination angle of the rotated beam. Details of the trepanning head they used are given in. ${ }^{6}$ It is worth mentioning that at pulse durations on a femtosecond time scale, material is removed much faster than heat can be conducted into the bulk substrate. Therefore, thermal effects or distortion of the surrounding area are minimized. $^{7)}$

The rotation of the direction of linearly polarized laser light during laser drilling can additionally improve the quality of the holes geometry. ${ }^{8,9)}$ Optical components like lenses and mirrors introduce astigmatism and change of the polarization leading to elliptical focal spots. Therefore, it is crucial to have 3D focal spot analysis before the installation of the trepanning head to prevent from irregular hole geometries with elliptical shapes.

After laser drilling the samples have been cross-sectioned along the hole axis to analyze the inner surface. The roundness of the hole on both entrance and exit sides show deviations of less than $2 \mu \mathrm{m}$, which is satisfactory within the demands of the automotive industry (Fig. 2). The exit hole laser beam exhibits better quality than the laser entrance hole. This can be ascribed to the shorter exposure time to the laser beam. In this study Kling et. al. ${ }^{5}$ utilize a scanning method based on the shear force microscopy (SHFM) for imaging with sub-micrometer spatial resolution and surface roughness analysis on the prepared samples. Results by the SHFM topography show a homogenous surface of the drilling hole (Fig. 3). The curvature in Fig. 3 (a) has to be removed, in order to measure the real roughness of the surface. The curvature is, firstly, estimated by using image manipulation software and then is removed as the topography surface promoted over a flat plane (Fig. 3 (b)). SHFM graph is analyzed by the root mean-square surface roughness ${ }^{R} Q$ as illustrated in Fig. 6. The evidence of a uniform surface texture is presented with only slight striations in the entrance and exit edges. At the entrance waviness
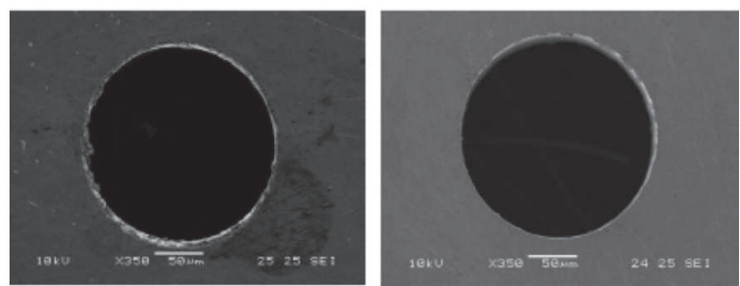

Fig. 2 Entrance side (left) and exit (right) of cylindrical hole of $175 \mu \mathrm{m}$ with a tolerance of less than $3 \%$. Kling et.al. ${ }^{5)}$

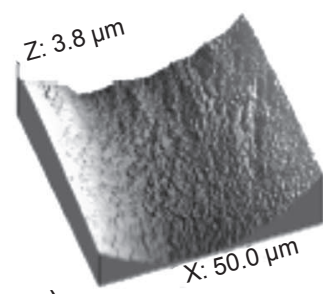

a)

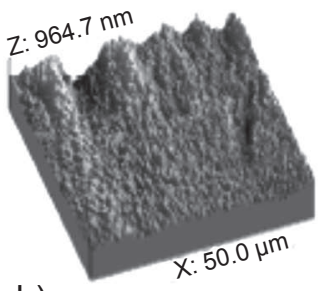

b)
Fig. 3 Topography map of the entrance area of the hole: (a) pseudo 3D topography (b) pseudo 3D topography after parabolic filtering to remove the hole curvature. Kling et. al. ${ }^{5)}$

is presented, giving maximum values of $160 \mathrm{~nm}$. In the central area the presence of peaks and valleys is uniform for different positions within the material thickness. At the exit zone it increases again.

In a similar work to that of Kling et. al., laser trepanning results on stainless steel, copper and carbon steel are shown by using a compact and inexpensive microchip laser fiber amplifier system $(100 \mathrm{ps}, 100 \mathrm{kHz}$, up to $80 \mu \mathrm{J}){ }^{10)}$ Although the overall precision of the structures is not as good as in the femtosecond regime, it is still satisfactory for many industrial standards. The fiber laser system Ancona et. al. suggests is a very attractive tool for a wide range of industrial applications where bulky and expensive regeneratively amplified modelocked picosecond lasers are used.

Recently, Moorhouse ${ }^{11)}$ demonstrated the advantages of ultrafast picosecond lasers in helical drilling for applications in fuel injection nozzles. The reduced thermal effects of the 10 ps pulse duration are also substantial to produce high quality drilled holes with smaller hole sizes $(\sim 100 \mu \mathrm{m})$ through 1 $\mathrm{mm}$ steel as illustrated in Fig. 5, where a comparison between EDM and laser drilled holes is performed. By keeping the laser fluence well above the laser ablation threshold, very high precision can be achieved. Furthermore, the developments of helical drilling optics that rotate the laser beam allow control of the hole taper. Hence, the freedom to form different nozzle shapes can be achieved by helical drilling. Nozzle shapes such as taperless or reversed taper holes as indicated in Fig. 6. It is possible to control the taper and to come up with cylindrical profiles by inclining the beam at small angles. Negative conicity holes can also be achieved by further inclining the beam during the circular motion, according to Kraus et. al. ${ }^{12)}$

Another recent research in hellical drilling technology, with the beam rotating like a conventional drill, has been demonstrated. Novel supermicro fibers based on cellulose and cellulose- 2.5- acetate have been made possible at the Institute of Textile Chemistry and Chemical Fibers ITCF in Germany as the result of the laser drilling of superfine spinnerets through which these new fibers can be extruded. ${ }^{13)}$ A new wet spinning technology based on these superfine spinnerets, which

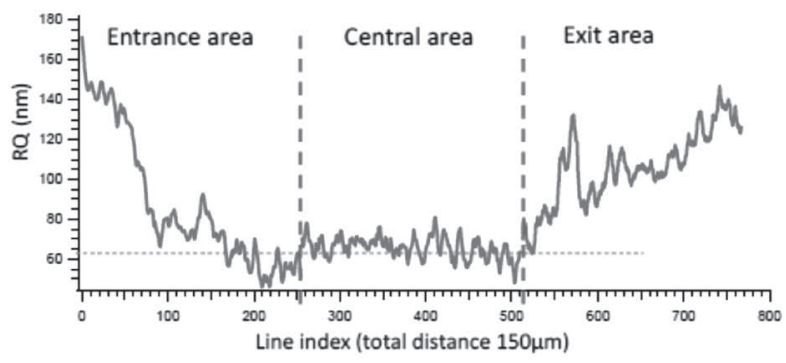

Fig. 4 Evolution of root-mean-square surface roughness inside the laser drilled hole. Kling et.al. ${ }^{5)}$ 


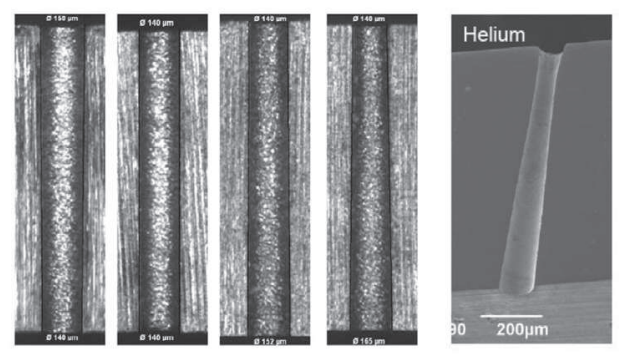

Fig. 5 Comparison of holes drilled in $1 \mathrm{~mm}$ thick steel by EDM (left) and picosecond laser (right). The laser drilled holes are narrower with cleaner sidewalls. Moorhouse. ${ }^{11)}$

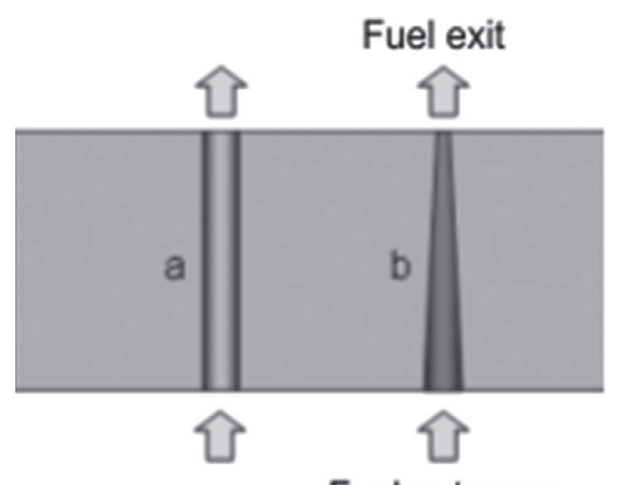

\section{Fuel entrance}

Fig. 6 Diesel injection nozzle shapes possible by helical drill optics: (a) taperless hole and (b) reverse taper holes. Moorhouse. ${ }^{11)}$

have approximately 2000 channels over the area of the globe on a one euro coin has been developed (Fig. 7). A large variation of spinneret materials and spinneret hole dimensions is possible and the outlets of the spinnerets are especially fine and smooth.

Since 2009 Bosch company performs micro-structuring on the injector of common rail diesel systems by using ultrafast laser pulses as illustrated in Fig. 8. A drained groove allows a tight system even at higher pressures of up to 2000 bar, leading to an even more reliable and environmentally-friendly diesel injection systems. ${ }^{14)}$

\subsection{Lubrication reservoirs in laser honing}

Another interesting material removal process where the ad-

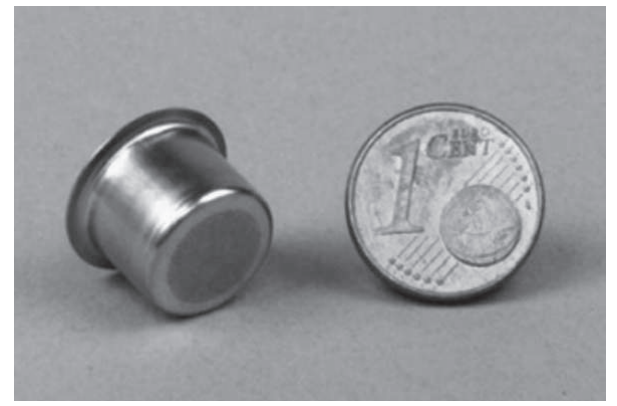

Fig. 7 A new wet spinning technology based on superfine spinnerets, which have approximately 2,000 channels over the area of the globe on a one euro. ${ }^{\dagger 2}$

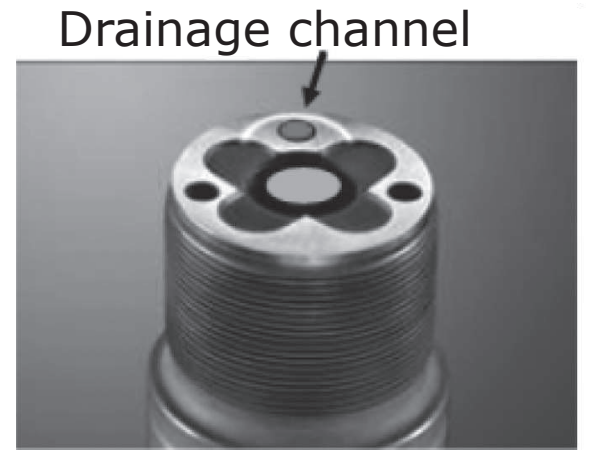

Fig. 8 In the seal face of the injector body a drainage channel is structures around the high pressure passage by ultrashort laser pulses. ${ }^{14)}$

vantages of ultrafast laser pulses for the automotive industry are obvious is the machining of lubrication reservoirs during laser honing. Due to strict exhaust and emission laws, decrease of the oil and fuel consumption of gasoline and diesel engines is required. The friction loss within the engine is a major factor in pushing the fuel consumption. The key for reducing friction in a piston/cylinder system is lubrication and surface texturing. Laser honing is a precision stock removal process which derives from the combination of a conventional honing process and laser texturing technology. It is still the leading one in cylinder texturing with notable effects on friction reduction. At early ' 90 s Kling and Grimm et. al.within Gehring $\mathrm{GmbH}$ invented laser honing technique and first applied it into processing the surface of a cylinder. In addition to the reduction of the wear of the cylinder and piston ring by $50 \%$, they reduced the oil consumption by $40 \%{ }^{\dagger 1}$ In a similar work Zhang et. al. ${ }^{15)}$ dropped the oil consumption by $53 \%$. In addition, Gehring company exhibits bore accuracy of less than $1 \mu \mathrm{m}$ diameter tolerance and surface precision of less than $0,04 \mu \mathrm{m}$ with ultrafast laser honing. Finish honing is used to remove the ejected molten mass arising during the laser processing and to produce an extremely fine sliding surface as shown in the upper right image of Fig. 9, comparing with the image on the bottom.

According to Abeln and Gehring company, the ideal lubrication conditions must be found. On the one hand, a high load capacity and therefore, a very good sliding property of the component should be come by low roughness, in other
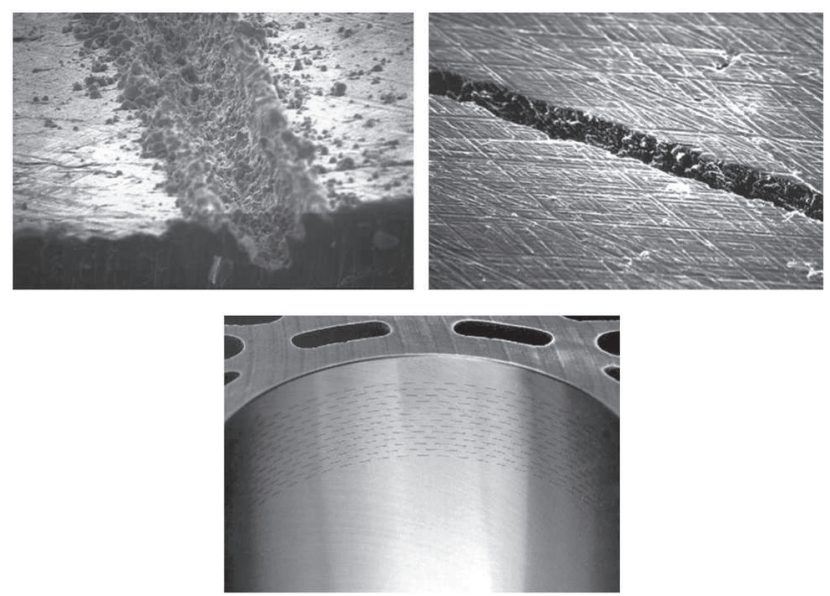

Fig. 9 Cylinder bore after laser honing. Gehring GmbH. ${ }^{1)}$

\footnotetext{
${ }^{\dagger 1}$ http://www.Gehring.de

${ }^{\dagger 2}$ http://www.innovationintextiles.com/2000-holes-on-the-euros-globe
} 

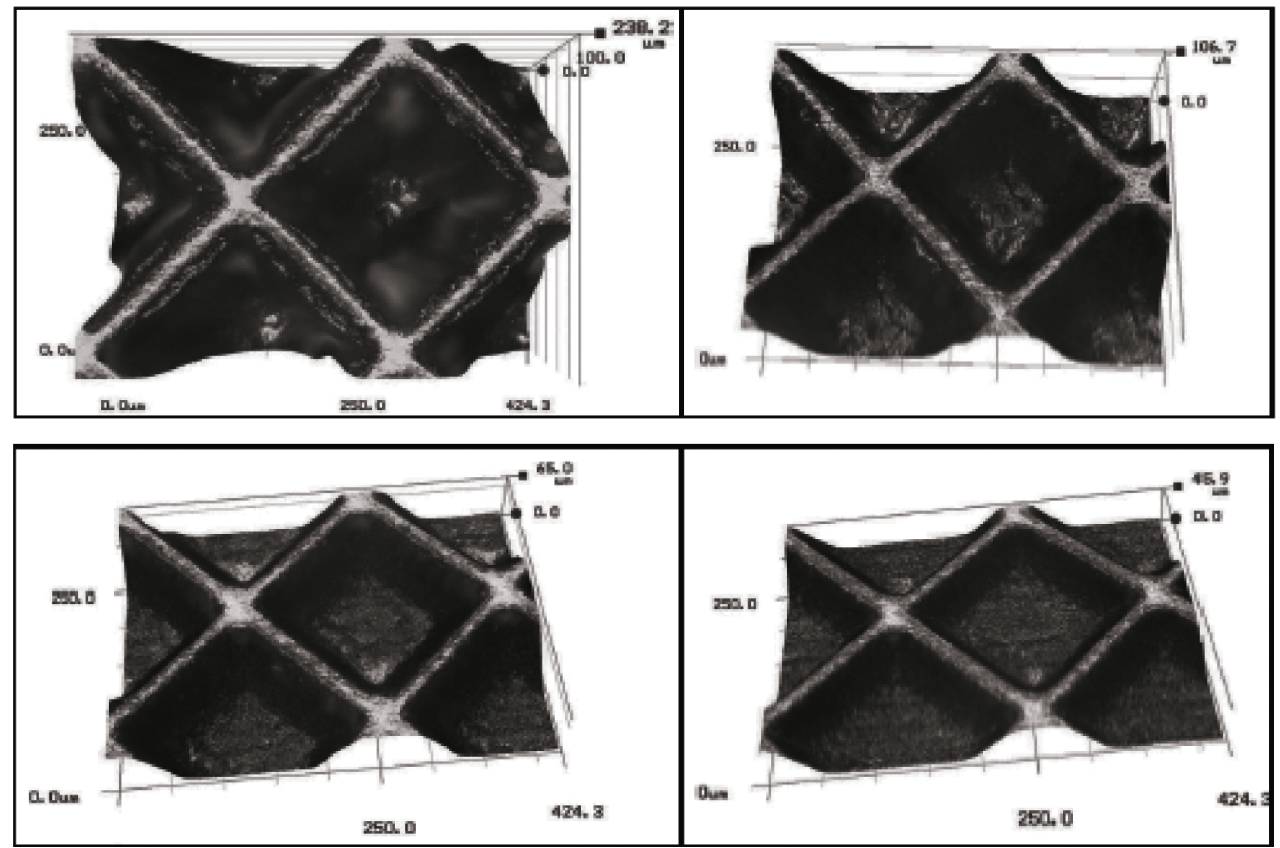

Fig. 10 Up: Scan velocity $3 \mathrm{~m} / \mathrm{s}$, pulse overlap 75\% (left). $12 \mathrm{~m} / \mathrm{s}$, pulse overlap 50\% (right). Down: $18 \mathrm{~m} / \mathrm{s}$ with pulse overlap 25\% (left) and $24 \mathrm{~m} / \mathrm{s}$ with pulse overlap $0 \%$ (right). Bruening et. al. ${ }^{17)}$

words, low depth of cuts. On the other hand the demand of an open structure is necessary so as to ensure wetting of the lubricant, which means roughness of the surface. This problem is resolved by placing defined oil retention pockets on a surface with low roughness ${ }^{16)}$ by using sub-100 picoseconf laser pulses. ${ }^{17)}$ Gehring reported that its lubrication-in-laser-honing technique has been successfully put into practice since January 2002, where it has been used in four-cylinder diesel engines. The experimental researches show that laser texturing not only can reduce the cost and the oil consumption of the engine, but also can prolong its operational life time.

\section{Mechanical industry}

Ultrafast laser pulses offer several advantages in the precision mechanical industry (e.g. in roll texturing). Further, high power ultrashort laser pulses are a powerful tool for 3D microstructuring of metal surfaces for printing applications. However, the technology of nanosecond lasers is still being preferred in the mechanical industry because of the low productivity of the ultrafast technologies due to the smaller ablation rate. On the other hand, nanosecond ablation is a fast, but not precise method comparing with picosecond and femtosecond lasers. A possibility to demonstrate very high ablation qualities is to avoid the accumulation of defects on the surface. This could be demonstrated by processing layer by layer with the same high quality. Recently, high scan speeds $(100 \mathrm{~m} / \mathrm{s})$ have been evaluated by Bruening et. al. for embossing and printing applications. ${ }^{18)}$ In order to achieve a layer-by-layer process, in other words pulse separation, Bruening et. al. use two different scanning techniques by using ps pulses with $2 \mathrm{MHz}$ repetition rate and $80 \mathrm{~W}$ output power.

Initially, they demonstrate pulse separation by a high-speed rotating work-piece, with the laser spot moving by a slow feed along the axis of a fast rotating cylinder. The rotational symmetry of the cylinder allows stable high scanning speeds of up to $40 \mathrm{~m} / \mathrm{s}$. By changing the rotation speed of the cylinder, the interaction between two consecutive pulses can be adjusted, so the pulse overlap can easily be avoided. In this way, accu- mulation of many pulses and high- precision can be achieved at the same time.

The second technique involves a combination of cylinder rotation and perpendicular scanning. This approach guarantees high speed and precision ablation process with repetition rates higher than $10 \mathrm{MHz}$. An algorithm was tested using a fast scanned laser spot and a slowly rotating cylinder, in order to deliver the sequence of pulses spatially on the cylinder surface. In the present set-up they used an AOD device, which allows to move the laser spot with feed rates above $100 \mathrm{~m} / \mathrm{s}$. During the ablation process, different scanning speeds (3-24 $\mathrm{m} / \mathrm{s}$ ) have been utilized and different fluences by using an external pulse picker $\left(0.5-13 \mathrm{~J} / \mathrm{cm}^{2}\right)$. The results of this investigation indicate that for lower scanning speeds with high pulse overlap an increased ablation rate has been observed, caused by an enhanced pulse to pulse interaction. Different materials (nickel. copper, steel and stainless steel) exhibit the same behavior. In addition, the pulse overlap has a significant influence on the quality of the process materials as illustrated in Fig. 10.

\section{Conclusion}

In the automotive industry, the advantages of ultrafast laser pulses together with trepanning system, helical drilling and polarization control promise micro machining with very high precision, repeatability of the drilling process, and high quality of the entrance and exit holes with negligible thermal effects. Using the honing technology, environmental impact is minimized. Laser honing is a proper method for finishing the inner walls of the bores for an internal combustion engine, while the lubrication of the cylinder and the wear-resistance properties can be improved. Concluding, the pulse-to-pulse overlap has a significant impact on both, quality and productivity in roll texturing applications.

\section{References}

1) C. Momma, B. N. Chichkov, S. Nolte, F. von Alvensleben, A. 
Tuennermann and H. Welling: Opt. Commun. 129 (1996) 134.

2) H. K. Toenshoff, F. von Alvensleben, A. Ostendorf, G. Kamlage and S. Nolte: IJEM (Review-International Journal of Electrical Machining) 4 (1999) 1.

3) H. Morita,T.Yamaguchi and N. Mohri: Journal of the JSEME 44 (2010) 45.

4) G. Kamlage, T. Bauer, A. Ostendorf and B. N. Chichkov: Appl. Phys. A 77 (2003) 307.

5) R. Kling, M. Dijoux, L. Romoli, F. Tantussi, J. Sanabria and E. Mottay: Proc. SPIE 8608 (2013).

6) D. Ashkenasi, N. Mueller, T. Kaszemeikat and G. Illing, Proc. LPM2010 (2010) 1.

7) M. D. Perry, B. C. Stuart, P. S. Banks, M. D. Feit, V. Yanovsky. and A. M. Rubenchik: J. Appl. Phys. 85 (1999) 6803.

8) S. Nolte, C. Momma, G. Kamlage, A. Ostendorf, C. Fallnich, F. von Alvensleben and H. Welling: Appl. Phys. A 68 (1999) 563.

9) H. K. Toenshoff, C. Momma, A. Ostendorf, S. Nolte and G. Kamlage: Journal of Laser Applications 12 (2000) 1.
10) A. Ancona, D. Nodop, J. Limpert, S. Nolte and A. Tuennermann: Appl. Phys. A 94 (2009) 19.

11) C. Moorhouse: Phys. Procedia 41 (2013) 374.

12) M. Kraus, S. Collmer, S. Sommer, A. Michalowski and F. Dausinger: Proc. Fourth Int. WLT Conf. Lasers in Manufacturing, Munich (2007) 639.

13) D. Ingildeev, F. Hermanutz, M. R. Buchmeiser, V. Onuseit, A Feuer, and R. Weber: 23. Stuttgarter Kunststoff-Kolloquium, Stuttgart (2013).

14) J. Koenig and T. Bauer: Proc. of SPIE 7925 (2011).

15) Y. Zhang, C. Yang, Y. Fu, J. Zhou, X. Hua, and J. Ji: Key Engineering Materials, 359-360 (2008) 340.

16) H. Gehring and T. Abeln: Diesel \& Gas Turbine World Wide, May (2008) 46.

17) T. Abeln and O. Kull: Patent No 20080308750 (2008).

18) S. Bruening, G. Hennig, S. Eifel, and A. Gillner: LiM 2011 Phys. Procedia 12 (2011) 105. 